\title{
CRITÉRIOS DE ESCOLHA PORTUÁRIA: UMA REVISÃO SISTEMÁTICA DA LITERATURA
}

\section{PORT SELECTION CRITERIA: A SYSTEMATIC REVIEW OF THE LITERATURE}

\author{
Guilherme Bergmann Borges Vieira ${ }^{1}$; Roberto Birch Gonçalves ${ }^{2}$ Fernanda Lazzari $^{3}$; Sarah Bertuol ${ }^{4}$ \\ ${ }^{1}$ Universidade de Caxias do Sul - UCS - Rio Grande do Sul \\ gbbvieir@ucs.br \\ ${ }^{2}$ Universidade de Caxias do Sul - UCS - Rio Grande do Sul \\ rbgoncal@ucs.br \\ ${ }^{3}$ Universidade de Caxias do Sul - UCS - Rio Grande do Sul \\ flazzar3@ucs.br \\ ${ }^{4}$ Universidade de Caxias do Sul - UCS - Rio Grande do Sul \\ sarahbertuol@hotmail.com
}

\begin{abstract}
Resumo
Com a globalização e desenvolvimento do comércio internacional, a concorrência entre os portos tem se intensificado e estudos portuários tem se desenvolvido. Parte desses estudos aborda a escolha portuária pela perspectiva dos diferentes atores envolvidos. Dado esse contexto, este artigo apresenta uma revisão sobre os critérios de escolha portuária com base nas perspectivas de armadores, agentes de carga e embarcadores. A revisão sistemática foi a técnica escolhida por proporcionar uma análise sistematizada e consistente da literatura existente sobre o tema, esclarecendo os principais critérios para a seleção de portos identificados em estudos anteriores e evidenciando aspectos convergentes e divergentes dos artigos selecionados. A contribuição da presente revisão sistemática baseia-se nos seguintes aspectos: identificação dos principais autores que estudaram o tema; principais instituições a que estão afiliados; principais periódicos utilizados para a apresentação dos resultados das pesquisas; número de publicações por país; número de publicações por ano e identificação dos critérios de escolha portuária, considerando as perspectivas dos diferentes atores envolvidos.
\end{abstract}

Palavras-chave: escolha portuária; critérios; literatura; revisão sistemática.

\section{Introdução}

O presente estudo trata-se de uma revisão sistemática da literatura sobre critérios de escolha portuária. Como o próprio nome sugere, essa técnica é utilizada para uma análise sistematizada e replicável de literatura selecionada a partir de uma questão claramente formulada.

A revisão sistemática gira em torno de estudos que tenham sido realizados com o mesmo propósito ou questão-chave, ou seja, agrupam o máximo de informações similares sobre um mesmo tema. Pode-se definir esse método como uma "síntese rigorosa de todas as pesquisas relacionadas com uma questão específica" (GALVÃO; SAWADA; TREVIZAN, 2004, p. 550). 
As revisões sistemáticas de literatura são comuns na área da saúde em momentos de busca de tratamentos e curas, ou até mesmo de padronizações de procedimentos (DE-LA-TORREUGARTE-GUANILO; TAKAHASHI; BERTOLOZZI, 2011). Ainda, ao viabilizarem, de forma clara e explícita, um resumo de todos os estudos sobre determinada intervenção, as revisões sistemáticas permitem incorporar um espectro maior de resultados relevantes, ao invés de limitar as conclusões à leitura de somente alguns artigos (SAMPAIO e MANCINI, 2006). Muitas barreiras são encontradas quando é desenvolvida uma revisão tradicional de literatura, pois a mesma agrega diferentes evidências e suposições a partir de um foco predeterminado pelo autor e segundo referências por ele escolhidas. Por esses motivos, entre outros, é que se indaga a confiabilidade e veracidade dos resultados encontrados.

Como alternativa a esse problema de baixa confiabilidade das revisões tradicionais de literatura, pesquisadores que buscam o saber de um tema específico diante de um problema em questão podem se utilizar de uma revisão sistemática da literatura, a qual agrupa diversos estudos voltados a um mesmo tema, constituindo assim uma conclusão mais sólida e confiável. Esse tipo de revisão demanda maior detalhamento e cuidado no afunilamento de dados comprobatórios de pesquisa.

Quando se encontram muitos estudos que abordam o mesmo assunto, há necessidade de filtrar as informações semelhantes, possibilitando identificar as respostas que apresentem opções mais viáveis diante do problema de pesquisa. O agrupamento de resultados aumenta a confiabilidade das conclusões a respeito da literatura revisada.

Segundo Cordeiro (2007, p. 428), “o grande volume das informações científicas aponta para a necessidade de sínteses que facilitam o acesso às mesmas, possibilitando conclusões baseadas na combinação dos resultados oriundos de múltiplas fontes". É nesse contexto que se insere a revisão sistemática, a qual pode ser definida como "um tipo de investigação científica que tem por objetivo reunir, avaliar criticamente e conduzir uma síntese dos resultados de múltiplos estudos primários" (CORDEIRO, 2007, p. 249), ou seja, trata-se de uma absorção de informações referentes a uma questão claramente formulada, por meio de uma síntese da literatura de estudos anteriores, desenvolvidos por diferentes autores, direcionados a um mesmo tema e que apresentam resultados como maior ou menor grau de convergência.

Segundo Sampaio e Mancini (2006, p. 83), "boas revisões sistemáticas são recursos importantes ante o crescimento acelerado da informação científica”, onde a relevância de analisar e agrupar a qualidade dos estudos selecionados torna-se essencial na busca de uma única conclusão, conduzindo ao 'estado da arte' e apontando para os limites do conhecimento escrito numa determinada área (LEVY e ELLIS, 2006). 
A revisão sistemática pode ser realizada com ou sem meta-análise que, segundo Sampaio e Mancini (2006, p. 83-89) trata-se de "um estudo de revisão da literatura em que os resultados de vários estudos independentes são combinados e sintetizados por meio de procedimentos estatísticos". Nos casos em que a meta-análise também é aplicada, o sucesso nem sempre persiste, devido à má qualidade metodológica do que já foi realizado, ou em função dos distintos procedimentos utilizados. Em função dessa dificuldade, no presente estudo optou-se por uma revisão sistemática sem meta-análise.

A primeira revisão sistemática que se tem conhecimento foi realizada em 1904 com base em apenas dois estudos. Após ser introduzida como base de conhecimento da ciência, foi aplicada constantemente nos anos 80 na prática clínica. Recentemente, vem sendo realizada com foco na gestão, produção e operações, descaracterizando seu uso como uma particularidade das ciências médicas. No âmbito da logística, a revisão sistemática pode atuar como simplificadora no agrupamento de informações importantes em diferentes contextos. No caso das decisões logísticas e, especialmente, da escolha portuária, tal técnica pode ser útil para a identificação dos critérios considerados pelos diferentes atores envolvidos no processo de seleção de portos: armadores, embarcadores (exportadores e importadores) e agentes de carga.

Neste estudo, o tema escolhido para a realização da revisão sistemática foi a escolha portuária, especificamente os critérios de escolha portuária. Buscou-se na revisão sistemática analisar e qualificar os estudos sobre escolha portuária realizados de 2000 a 2011. Para tanto, foram consultadas todas as publicações sobre o tema disponíveis nas bases de dados ScienceDirect e Scopus, considerando também critérios de inclusão e exclusão previamente determinados, definidos pela questão norteadora do estudo.

O estudo baseou-se nas seguintes perguntas: i) Como evoluíram as pesquisas sobre escolha portuária no período de 1990 a 2011? ii) Quais os critérios de escolha portuária considerados nesses estudos? iii) Quais os tipos de amostra utilizados (armadores, agentes de carga, exportadores, importadores)? iv) Quais as técnicas de análise utilizadas? v) Quais os resultados obtidos? Para responder a essas perguntas, foi estabelecido o objetivo geral de 'analisar a evolução das pesquisas sobre escolha portuária no período de 1990 a 2011'. No intuito de operacionalizar o alcance do objetivo geral, foram estabelecidos os seguintes objetivos específicos: i) apresentar a metodologia de revisão sistemática da literatura; ii) selecionar estudos sobre escolha portuária desenvolvidos no período analisado; iii) identificar as abordagens utilizadas nos estudos; e iv) propor sugestões de novos estudos sobre o tema a partir das análises efetuadas.

A escolha portuária é um fator importante nas operações de exportação e importação, podendo afetar tanto os custos logísticos como o nível dos serviços, influenciando outras variáveis de decisões empresariais (THOMAZ e SERRA; MARTINS e BRONZO, 2009). Tanto os pontos 
positivos quanto os negativos de um sistema portuário podem afetar todo o comportamento logístico das transações internacionais e, portanto, a escolha portuária influencia diretamente o desempenho dos transportadores e usuários (NIR; LIN; LIANG, 2003). Através desta pesquisa e das conclusões proporcionadas pela mesma, busca-se auxiliar os diferentes atores participantes da cadeia logístico-portuária, tais como gestores portuários, armadores, agentes de carga, exportadores e importadores na compreensão dos critérios utilizados para a escolha de portos em operações internacionais.

\section{Revisão sistemática}

Observando os números de estudos que são publicados a cada ano, é possível perceber que os estudos de revisão tornaram-se um desafio para os profissionais e cientistas em geral (PIEPER et al, 2012). Nos últimos 30 anos, as sínteses de pesquisas têm se desenvolvido sob a forma de revisões sistemáticas para lidar com a quantidade sempre crescente de pesquisa primária. Hoje em dia, tornou-se difícil inclusive manter o controle do número de revisões sistemáticas que estão sendo produzidos e este aumento tem levado os revisores à realização de revisões sistemáticas de revisões sistemáticas, o que vem gerando um novo tipo de publicação (PIEPER et al, 2012).

Para Galvão, Sawada e Trevizan (2004, p.550), a revisão sistemática pode ser definida como "uma síntese rigorosa de todas as pesquisas relacionadas com uma questão específica". Segundo Sampaio e Mancini (2006, p. 84), “uma revisão sistemática, assim como outros tipos de revisão, é uma forma de pesquisa que utiliza como fonte de dados a literatura sobre determinado tema". Em outras palavras, pode-se definir esse tipo de pesquisa como uma análise secundária acerca dos estudos primários realizados sobre um mesmo tema.

Lima, Soares e Bacaltchuk (2000) definem revisão sistemática como uma síntese das informações disponíveis em dado momento sobre um problema específico, de forma objetiva e reproduzível, por meio de método científico, tendo como princípios gerais a exaustão na busca dos estudos analisados, a seleção justificada dos estudos por critérios de inclusão e exclusão explícitos e a avaliação da qualidade metodológica. Segundo os autores, uma revisão sistemática de literatura pode incluir também uma quantificação por meio de técnicas estatísticas (meta-análise).

O objetivo de se realizar uma revisão sistemática é agrupar o conhecimento e pesquisa de diversos autores, com definições e conclusões similares ou divergentes, associando as diferentes informações com o propósito de gerar uma só conclusão. Esse tipo de síntese propicia a oportunidade de fornecer aos estudiosos e pesquisadores um amplo resumo de provas, permitindo a comparação de múltiplas conclusões e o contraste de diferentes achados não necessariamente convergentes. As razões para discordância podem incluir populações diferentes, projetos de estudo diferentes ou resultados e interpretações diferentes dos mesmos dados. Nos casos de discordância, a 
identificação das razões para resultados divergentes permite que os usuários baseiem suas decisões sobre a evidência mais confiável e adequada a cada situação particular (PIEPER et al, 2012).

Sampaio e Mancini (2006) apresentam cinco etapas essenciais na construção da revisão sistemática de literatura: i) a definição da pergunta; ii) a busca das evidências; iii) a seleção dos estudos; iv) a análise dos estudos; e v) a apresentação dos resultados.

Primeiramente, deve-se definir uma pergunta-chave, científica, onde se deve especificar a intervenção de interesse do pesquisador. Para haver uma análise consistente, deve-se elaborar uma pergunta clara e objetiva. As revisões sistemáticas visam identificar todos os estudos sobre uma questão específica, de modo a gerar um resumo equilibrado e imparcial da literatura (NIGHTINGALE, 2009), tornando a questão explorada peça-chave para a obtenção dos resultados finais.

Quanto à busca das evidências, pode ser encontrada uma imensa variedade de artigos tanto em bases de dados como em outras fontes, tais como bancos de teses, dissertações e estudos monográficos (gray literature). No entanto, as bases de dados caracterizam-se como as principais fontes na busca de artigos e estudos relacionados ao tema em questão.

No que se refere à seleção dos estudos, tendo como base a pergunta inicial, devem ser estabelecidas estratégias de busca a partir de termos-chave de pesquisa, considerando também critérios de inclusão e exclusão previamente definidos. Isso permite que se mantenha o foco no objeto de estudo e se evitem problemas decorrentes da miscigenação de temas. Em suma, trata-se de obter evidências relevantes para a conclusão final, seguindo as estratégias escolhidas no trajeto de pesquisa e formalizadas em um protocolo de revisão sistemática. Títulos e introduções bem formulados são importantes no período de escolha, pois tendem a serem esclarecedores.

Com os estudos selecionados, parte-se para a análise do conteúdo apresentado em cada estudo, chegando-se na apresentação dos resultados encontrados. Essa etapa é a mais importante, pois agrupa o conhecimento desenvolvido em todos os artigos revisados, identificando concordâncias e divergências que ajudam a responder a questão-chave, a qual norteia toda a pesquisa.

É importante salientar que, para que uma revisão sistemática seja considerada correta e adequada, devem-se seguir estritamente os critérios de seleção de artigos estabelecidos, de modo que sejam encontrados os mesmos resultados se a revisão for realizada novamente por diferentes pesquisadores.

\section{Escolha portuária}

Os portos são definidos por Valentine e Gray (2002) como organizações complexas com uma diversidade de entradas e saídas, consubstanciada em diferentes aspectos físicos, de acesso, 
logísticos e legais, o que torna difícil o estudo e a comparação direta entre portos aparentemente homogêneos. Já para Bichou e Gray (2004), os portos são organizações multipartes e complexas nas quais instituições e funções normalmente se cruzam em vários níveis. Segundo Tolley e Turton (1995), os portos são um componente da distribuição física de mercadorias e oferecem uma interface marítimo-terrestre para atividades de exportação e de importação. Essa interface marítimoterrestre está diretamente relacionada com o processo de escolha portuária, o qual envolve diferentes atores da cadeia logística: i) os armadores, que definem os portos de escala em cada rota; ii) os embarcadores (exportadores/importadores), que definem os portos de embarque e desembarque em suas operações; e iii) os agentes de carga, que apoiam e auxiliam os exportadores e importadores no processo de escolha portuária.

No caso dos exportadores e importadores, a escolha portuária é condicionada pela modalidade de venda acordada entre as partes (Incoterm). Por exemplo, no caso de uma operação realizada sob o Incoterm Ex Works (EXW), o importador define e se responsabiliza por todas as formalidades da exportação. Nessa situação, a obrigação é mínima para o vendedor (exportador). Um exemplo inverso ocorre quando se utiliza o INCOTERM Delivered at Place (DAP). Nesse caso, a responsabilidade do exportador inclui a escolha dos portos que serão utilizados, tanto no país de origem quanto no de destino.

No que se refere aos critérios de escolha portuária, percebe-se que os mesmos variam de acordo com a perspectiva do ator a ser considerado. Os critérios considerados na escolha portuária são diversos e podem ser classificados em dois tipos: os fatores quantitativos e os fatores qualitativos. Os fatores quantitativos são aqueles que podem ser potencialmente medidos e comparados de forma objetiva, e são agrupados em três grandes categorias: i) fatores de rota; ii) fatores de custo; e iii) fatores de serviço (D'ESTE e MEYRICK, 1992). Já os fatores qualitativos incluem influências subjetivas, como flexibilidade e facilidade de uso, esforços de marketing do porto, tradição, contatos pessoais e nível de cooperação que podem ser desenvolvidos entre o usuário e o porto.

$\mathrm{Na}$ prática, a distinção entre os fatores quantitativos e qualitativos é turva porque a percepção do nível de desempenho do porto pode não ser um reflexo justo do desempenho real. Em muitos casos, as percepções podem ter precedência sobre performances reais (D'ESTE e MEYRICK, 1992). De qualquer modo, a performance portuária é um elemento do processo de escolha portuária realizado pelos diferentes atores da cadeia logística.

Quando considerada a perspectiva dos agentes de carga, podem-se citar os seguintes critérios de escolha portuária: i) frequência de visitas de navios; ii) eficiência; iii) adequação de infraestrutura portuária; iv) localização; v) tarifas portuárias; vi) resposta às necessidades dos usuários do porto; e vii) reputação por danos na carga (TONGZON, 2002). 
Quando a escolha portuária é efetuada pela perspectiva dos embarcadores, tanto exportadores quanto importadores, o pressuposto fundamental é de que, optando por um agente de cargas, implicitamente o embarcador acaba escolhendo um porto. No competitivo mercado de transporte são os atributos do serviço porta a porta que um agente oferece que influenciam a escolha do embarcador (MALCHOW e KANAFANI, 2004). Em outras palavras, no processo de escolha portuária, o embarcador é influenciado pelos atributos de serviço oferecidos pelo agente de cargas. Portanto, o processo de escolha portuária por parte dos embarcadores está inserido no processo de seleção de um agente de cargas que oferece um serviço através do porto em questão.

Já para os armadores, a preferência no momento da escolha portuária se mantém voltada aos serviços e instalações portuárias, além da localização geográfica, fator crítico para a definição de rotas, em função do maior ou menor potencial gerador de cargas do porto e do distanciamento do mesmo em relação a uma rota marítima principal (TIWARI; ITOH; DOI, 2003; VIEIRA e PASA, 2005).

Pode-se observar, portanto, que os critérios de escolha dos portos podem variar consideravelmente de ator para ator, porém sempre há presença de similaridades. Por exemplo, para Lirn et al (2004), os critérios de escolha portuária englobam fatores como a localização geográfica, volume de carga envolvido, nível e prestação de serviço, infraestrutura e gestão. Os autores ainda destacam outros critérios como custo de combustível, integração entre os portos de origem e destino e instalações portuárias de ambos. Por outro lado, Tongzon (2009) menciona critérios como eficiência, frequência das escalas de navios, adequação da infraestrutura, localização geográfica, tarifas portuárias, rapidez de resposta às necessidades dos usuários e reputação relativa a danos nas cargas. Entre tantas possibilidades de critérios a serem considerados, o determinante principal está relacionado à eficiência e ao desempenho de um porto em relação à movimentação de cargas que pode suportar. A seleção correta de um porto para operacionalizar uma exportação ou importação deve ser tratada como um problema complexo que requer um processo de avaliação ampla. Os pontos positivos e negativos são características que diferem de um local a outro e nem sempre são fáceis de identificar.

Onut, Umut e Torun (2011) descrevem a escolha portuária como um problema de múltiplos critérios de escolha (CMMS). Em seu artigo, os autores destacam entre os critérios principais a localização geográfica, as características físicas do porto, o custo e a eficiência, entre outros fatores como volume de carga, capacidade, segurança e frequência de entregas.

Para os portos marítimos serem considerados competitivos e sustentáveis no longo prazo em um mercado mundialmente turbulento e extremamente competitivo, faz-se também necessária uma análise das estruturas organizacionais e práticas de gestão existentes. Como os portos estão constantemente evoluindo e tornando-se cada vez mais integrados e incorporados em suas cadeias 
de suprimento, a questão da garantia de práticas de gestão da qualidade torna-se um fator importante (ONUT; UMUT; TORUN, 2011).

Assim, os inúmeros fatores envolvidos na escolha portuária, tais como custos e tempos (fatores burocráticos, armazenamento, movimentação), facilidades logísticas internas e externas ao porto, transit time, confiabilidade, entre outros, precisam ser entendidos à luz dos mais diversos pontos de vista. Tais pontos de vista são esclarecidos em uma revisão sistemática apurada e eficiente. Essa clareza na compreensão desses fatores conduz a uma boa escolha portuária, contribuindo fortemente com a eficiência da gestão de cargas internacional e auxiliando todos os atores envolvidos.

\section{Procedimentos metodológicos}

O método escolhido para este estudo foi a revisão sistemática da literatura publicada sobre o tema abordado (critérios de escolha portuária). O primeiro passo foi a preparação de um protocolo de revisão. O protocolo tem como papel auxiliar o acompanhamento da revisão sistemática ao longo de suas etapas.

É importante ter como determinante o tipo de informação que se busca e onde encontrá-la, devendo o pesquisador ser bastante específico no tipo de dados que está procurando, pois essa é uma questão relevante na escolha da base de dados. Neste trabalho, foram utilizadas as bases de dados Science Direct e Scopus, e o período de análise selecionado foi de 1990 a 2011. Foi possível observar a importância do registro da data em que a pesquisa foi realizada, pois a inserção de novos artigos na base de dados é constante. $\mathrm{Na}$ análise em questão, foram considerados os resultados obtidos em consulta às referidas bases de dados no dia 12 de setembro de 2012.

$\mathrm{Na}$ utilização da revisão sistemática, para um maior redirecionamento das informações específicas de busca, utilizam-se critérios de inclusão e exclusão. Entre os critérios de inclusão estão aqueles que devem constar ou ser citados nos estudos encontrados, sendo os critérios de exclusão o oposto, ou seja, o que não deve constar nos resultados de pesquisa. No caso do presente estudo, como critérios de inclusão, foram escolhidas expressões alinhadas com o foco do estudo: port choice, port selection, criteria. Também foram consideradas algumas variações, tais como seaport choice e seaport selection. Dois passos foram seguidos na decisão dos critérios de exclusão. Em um primeiro momento, foram escolhidos termos significativamente inadequados à busca e, posteriormente, realizou-se uma análise aprofundada a partir dos resultados inicialmente encontrados, o que possibilitou selecionar somente os estudos apropriados e voltados especificamente aos critérios de escolha portuária. Em síntese, os critérios de exclusão iniciais incluíram termos específicos como air port, air lines, ferry, dry port e outros. Após essa primeira filtragem, foi realizada uma análise dos títulos, resumos e palavras-chave de cada artigo, sendo 
desclassificados aqueles que não possuíam o conteúdo específico analisado nesta revisão sistemática: os critérios de escolha portuária.

Nesta revisão sistemática foram realizadas duas rodadas em cada base de dados. Inicialmente, foram identificados 85 artigos na base de dados Scopus e 258 artigos na base de dados Science Direct, totalizando 343 estudos que corresponderam aos critérios de busca inseridos e suas variações. Feito isso, foram eliminados 14 artigos identificados como duplicados, ou seja, que estavam presentes em ambas as bases, reduzindo-se o total de estudos para 329. O passo seguinte foi a utilização dos termos de exclusão nas próprias bases de dados e a análise dos títulos, resumos e palavras-chave dos artigos, o que possibilitou a seleção apenas de artigos compatíveis e direcionados exclusivamente aos critérios de escolha portuária, resultando em um número final de 49 artigos, os quais foram considerados na revisão sistemática. Tais artigos foram agrupados em uma tabela-mãe, a qual possibilitou o detalhamento de cada estudo as partir dos critérios estabelecidos no protocolo de revisão e a observação, de uma forma sintetizada, dos aspectos divergentes entre os artigos. Os artigos foram classificados considerando aspectos bibliométricos (autores, afiliações, países, periódicos e ano de publicação) e aspectos de conteúdo (tipo de amostra utilizada e critérios de escolha portuária identificados).

\section{Resultados}

Nesta seção são apresentados os resultados obtidos, sendo os mesmos divididos em aspectos bibliométricos e aspectos de conteúdo dos artigos selecionados.

\subsection{Aspectos bibliométricos}

Foram encontrados ao todo 99 autores que contribuíram com os 49 artigos escolhidos. A Tabela 1 apresenta o numero de artigos por autor.

Tabela 1 - Número de artigos por autor

\begin{tabular}{ccc}
\hline Autor & $\mathbf{n}^{\mathbf{0}}$ de artigos & $\mathbf{\%}$ \\
\hline Chou & 6 & $12,2 \%$ \\
Tongzon & 4 & $8,2 \%$ \\
Notteboom & 4 & $8,2 \%$ \\
Garcia-Alonso & 3 & $6,1 \%$ \\
Ng & 3 & $6,1 \%$ \\
Musso & 2 & $4,1 \%$ \\
Chang & 2 & $4,1 \%$ \\
Lee & 2 & $4,1 \%$ \\
Wong & 2 & $4,1 \%$ \\
Ferrari & 2 & $4,1 \%$ \\
Sanchez & 2 & $4,1 \%$ \\
Veldman & 2 & $4,1 \%$ \\
Beresford & 2 & $4,1 \%$ \\
Low & 2 & $4,1 \%$ \\
Lam & 2 & $4,1 \%$ \\
Tang & 2 & $4,1 \%$ \\
Yeo & 2 & $4,1 \%$
\end{tabular}




\begin{tabular}{ccc} 
Roe & 2 & $4,1 \%$ \\
Dinwoodie & 2 & $4,1 \%$ \\
Outros & 1 & $2,0 \%$ \\
\hline Total & 49 & $100,0 \%$ \\
\hline
\end{tabular}

Entre os 99 autores, um autor publicou seis artigos; dois autores publicaram quatro artigos; dois autores publicaram três artigos; 14 autores publicaram dois artigos e 80 autores tiveram participação em apenas um artigo. Percebe-se, portanto, uma concentração relativamente pequena nos trabalhos selecionados nesta revisão sistemática.

A pesquisa identificou no total 63 Instituições relacionadas aos autores. Na Tabela 2 são apresentadas as 49 Instituições a que o primeiro autor de cada artigo estava afiliado.

\begin{tabular}{ccc}
\multicolumn{2}{c}{ Tabela 2} & Afiliação dos autores \\
\hline Afiliação & $\mathbf{n}^{\mathbf{0}}$ de artigos & $\mathbf{\%}$ \\
\hline Cardiff University & 2 & $4,1 \%$ \\
Erasmus University & 2 & $4,1 \%$ \\
Inha University & 3 & $6,1 \%$ \\
National Kaohsiung Marines University & 7 & $14,3 \%$ \\
Outros & 35 & $71,4 \%$ \\
\hline Total & 49 & $100,0 \%$ \\
\hline
\end{tabular}

A National Kaohsiung Marines University, Universidade de Taiwan, apresentou o maior número de artigos publicados, com um número total de sete estudos, seguida pela Inha University, da Coréia do Sul.

Os estudos selecionados estão presentes em um número total de 29 journals. O periódico Maritime Economics and Logistics representou a maior parte das publicações, com oito artigos e presença percentual em 16,3\% dos estudos, seguido pelo periódico Transportation Research Part A: Policy and Practice, com cinco artigos e participação em 10,2\% dos estudos, conforme apresentado na Tabela 3.

Tabela 3 - Principais periódicos

\begin{tabular}{lcc}
\hline Periódico & $\mathbf{N}^{\mathbf{0}}$ de artigos & $\mathbf{\%}$ \\
\hline Maritime Economics and Logistics & 8 & $16,3 \%$ \\
Transportation Research Part A: Policy and Practice & 5 & $10,2 \%$ \\
Research in Transportation Economics & 4 & $8,2 \%$ \\
Journal of Transport Geography & 3 & $6,1 \%$ \\
Maritime Policy and Management & 2 & $4,1 \%$ \\
The Asian Journal of Shipping and Logistics & 2 & $4,1 \%$ \\
Transportation Research Part E: Logistics and Transportation Review & 2 & $4,1 \%$ \\
Transport Policy & 2 & $4,1 \%$ \\
Outros & 21 & $42,9 \%$ \\
\hline Total & 49 & $16,3 \%$ \\
\hline
\end{tabular}

$\mathrm{Na}$ análise dos países de cada artigo publicado, destacaram-se a Coréia do Sul e Taiwan com seis artigos cada, conforme Tabela 4. Singapura e Holanda contribuíram com quatro artigos; China, Espanha, Itália e Reino Unido apresentaram três artigos; Estados Unidos e Austrália dois artigos; e os demais países apenas um artigo cada. Percebe-se que a concentração dos artigos selecionados nesta revisão sistemática nos países da Europa é significativa, com um total de 13 artigos (26,5\% do 
total) oriundos de países como Espanha, Itália, Holanda e Reino Unido. Todavia, o continente asiático destacou-se com o maior número de contribuições, destacando-se o número de publicações originadas na Coréia do Sul e em Taiwan.

Tabela 4 - Publicações por país

\begin{tabular}{lcc}
\hline País & $\mathbf{N}^{\mathbf{0}}$ de artigos & $\mathbf{\%}$ \\
\hline Austrália & 2 & $4,1 \%$ \\
Estados Unidos & 2 & $4,1 \%$ \\
China & 3 & $6,1 \%$ \\
Espanha & 3 & $6,1 \%$ \\
Itália & 3 & $6,1 \%$ \\
Reino Unido & 3 & $6,1 \%$ \\
Singapura & 4 & $8,2 \%$ \\
Holanda & 4 & $8,2 \%$ \\
Coréia do Sul & 6 & $12,2 \%$ \\
Taiwan & 6 & $12,2 \%$ \\
Outros & 13 & $26,5 \%$ \\
\hline Total & 49 & $4,1 \%$ \\
\hline
\end{tabular}

A pesquisa foi baseada nos artigos publicados entre os anos de 1990 e 2011. A Tabela 5 apresenta o número de publicações por ano.

\begin{tabular}{lcc}
\multicolumn{3}{c}{ Tabela 5 - Publicações por ano } \\
\hline Ano & $\mathbf{n}^{\mathbf{0}}$ de artigos & $\%$ \\
\hline 1995 & 1 & $2,0 \%$ \\
1998 & 1 & $2,0 \%$ \\
2001 & 1 & $2,0 \%$ \\
2002 & 1 & $2,0 \%$ \\
2003 & 1 & $2,0 \%$ \\
2004 & 1 & $2,0 \%$ \\
2006 & 6 & $12,2 \%$ \\
2007 & 2 & $4,1 \%$ \\
2008 & 3 & $6,1 \%$ \\
2009 & 6 & $12,2 \%$ \\
2010 & 10 & $20,4 \%$ \\
2011 & 14 & $28,6 \%$ \\
2012 & 1 & $2,0 \%$ \\
\hline 2013 & 1 & $2,0 \%$ \\
\hline Total & 49 & $100,0 \%$ \\
\hline
\end{tabular}

Conforme se pode observar na Tabela 5, dois artigos são datados de 2012 e 2013. Tratam-se de estudos em versão preliminar já disponíveis nas bases de dados. Devido à relevância desses artigos para o tema em estudo, os mesmos foram considerados na análise.

Dentre os anos selecionados, foram identificados mais artigos publicados no ano de 2011, com um total de 14, seguido de 10 artigos em 2010, representando quase a metade do total de artigos selecionados. Nos anos anteriores a 1995, nenhum artigo foi selecionado para a revisão. Isso sugere uma maior preocupação com o estudo do tema nos últimos anos. 


\subsection{Aspectos de conteúdo}

Nos aspectos de conteúdo, cada artigo foi categorizado de acordo com: i) o tipo de amostra (ator) considerado - embarcadores (exportadores e importadores), armadores ou agentes de carga; e ii) os critérios de escolha portuária identificados.

Quanto às amostras utilizadas nos estudos, notou-se uma predominância de artigos considerando a perspectiva do armador. Totalizando 27 artigos de 49 selecionados, os estudos que enfocam a perspectiva do armador, representam $55,1 \%$ do que foi analisado nesta revisão sistemática, seguidos por artigos que contemplam a perspectivas dos embarcadores $(24,5 \%)$ e artigos com a perspectiva dos agentes de carga (20,4\%).

Tabela 6 - Tipos de amostra considerados nos estudos

\begin{tabular}{lcc}
\hline Amostra & $\mathbf{n}^{\mathbf{0}}$ de artigos & $\mathbf{\%}$ \\
\hline Agente de carga & 10 & $20,4 \%$ \\
Armador & 27 & $55,1 \%$ \\
Embarcador & 12 & $24,5 \%$ \\
\hline Total & 49 & $20,4 \%$ \\
\hline
\end{tabular}

Quanto aos critérios de escolha portuária, foram identificados 24 fatores, os quais são apresentados na Tabela 7. A localização geográfica foi o critério mais citado, com participação de $71 \%$ no total de 49 artigos, ou seja, este critério está presente em 35 dos 49 artigos selecionados. A seguir, aparecem os critérios custos logísticos e qualidade dos serviços.

Tabela 7 - Critérios de escolha portuária

\begin{tabular}{lcc}
\hline Critérios & $\mathbf{n}^{\mathbf{0}}$ de artigos & $\mathbf{\%}$ \\
\hline Atendimento ao cliente & 1 & $2,0 \%$ \\
Volume de carga total & 1 & $2,0 \%$ \\
Capacidade de manuseio de documentos eletrônicos & 1 & $2,0 \%$ \\
Taxas portuárias & 1 & $2,0 \%$ \\
Segurança portuária & 1 & $2,0 \%$ \\
Frequência de embarques & 2 & $4,1 \%$ \\
Operações portuárias & 2 & $4,1 \%$ \\
Reputação por danos na carga & 2 & $4,1 \%$ \\
Custo de combustível & 2 & $4,1 \%$ \\
Tamanho do exportador & 2 & $4,1 \%$ \\
Capacidade operacional & 2 & $4,1 \%$ \\
Custo de transporte terrestre (inland) & 2 & $4,1 \%$ \\
Nível de produtividade & 3 & $6,1 \%$ \\
Capacidade de armazenagem & 4 & $8,2 \%$ \\
Tempo total de trânsito & 4 & $8,2 \%$ \\
Tamanho do porto & 4 & $8,2 \%$ \\
Fretes marítimos a partir do porto & 5 & $10,2 \%$ \\
Infraestrutura & 5 & $12,2 \%$ \\
Custos portuários & 6 & $12,2 \%$ \\
Eficiência & 6 & $12,2 \%$ \\
Conexões modais & 6 & $20,4 \%$ \\
Qualidade de serviço & 10 & $22,4 \%$ \\
Custos logísticos & 11 & $24,5 \%$ \\
Localização geográfica & 12 & $71,4 \%$ \\
\hline Total de artigos & 35 & $\mathbf{1 0 0 , 0 \%}$ \\
\hline
\end{tabular}


Percebe-se certo grau de sobreposição entre os critérios citados nos diferentes estudos, o que permite seu agrupamento em categorias. Por exemplo, os critérios taxas portuárias, custo de combustível, custo de transporte terrestre (inland), frete marítimos a partir do porto, custos portuários e custos logísticos, este último com uma abrangência bem maior que a dos demais, podem ser agrupados em um único fator denominado custos logísticos-portuários. Nesse sentido, os 24 foram critérios agrupados em quatro categorias: i) custos logístico-portuários; ii) nível de serviço; iii) características físicas/operacionais do porto e iv) características da operação de exportação/ importação. A Figura 1 apresenta os critérios associados a cada categoria, bem como o número de artigos em que são citados tais critérios.

Figura 1 - Agrupamento dos fatores em categorias

\begin{tabular}{|cc|ccc|}
\hline Custos logístico-portuários & $\mathbf{n}^{\mathbf{0}}$ art. & Características físicas/operacionais do porto & $\mathbf{n}^{\mathbf{0}}$ art. \\
Taxas portuárias & 1 & Volume de carga total & 1 \\
Custo de combustível & 2 & Capacidade de manuseio de documentos eletrônicos & 1 \\
Custo de transporte terrestre (inland) & 2 & Operações portuárias & 2 \\
Frete marítimos a partir do porto & 5 & Capacidade operacional & 2 \\
Custos portuários & 6 & Capacidade de armazenagem & 4 \\
Custos logísticos & 12 & Tamanho do porto & 4 \\
Nível de serviço & $\mathbf{n}^{\mathbf{0}}$ art. & 1 & Infraestrutura & 6 \\
Atendimento ao cliente & 1 & Conexões modais & 10 \\
Segurança portuária & 2 & Características da operação de exportação/ importação no art. \\
Reputação por danos na carga & 3 & Frequência de embarques & 2 \\
Nível de produtividade & 6 & Tamanho do exportador & 2 \\
Eficiência & 11 & Tempo total de trânsito & 4 \\
Qualidade de serviço & & Localização geográfica & 2 \\
\hline
\end{tabular}

A categoria 'custos logístico-portuários' contempla critérios citados em 28 artigos $(57 \%$ do total de artigos selecionados), destacando-se os custos logísticos (citados de forma abrangente) e os custos portuários, em termos mais específicos. Cabe salientar que os custos logísticos foram considerados em 12 artigos, enquanto os custos portuários foram encontrados em apenas seis. Isso sugere que os usuários, ao selecionarem os portos de embarque e desembarque, mais do que os custos portuários propriamente ditos, consideram os custos logísticos totais decorrentes de determinada escolha portuária. Isso porque os custos portuários (de origem e de destino) são apenas um elemento na composição do custo logístico total.

Quanto à categoria 'nível de serviço', os fatores mencionados estão presentes em 24 artigos (49\% do número total de artigos). Nessa categoria, merecem destaque os critérios 'qualidade do serviço' e 'eficiência'.

No que se referem à categoria 'características físicas/ operacionais do porto', nove critérios foram encontrados na amostra de artigos selecionados, merecendo destaque a localização 
geográfica, as conexões intermodais do porto e a infraestrutura disponível, mencionadas em 35, dez e seis estudos, respectivamente.

Finalmente, no que se refere às 'características da operação de exportação/ importação', o fator mais mencionado foi o tempo total de trânsito. Isso sugere que, ao selecionar os portos de embarque e desembarque, mais do que os aspectos portuários em si, os usuários consideram o contexto mais amplo do tempo de trânsito porta a porta, o que encontra eco na literatura. Os portos são um fator importante na composição desse tempo, mas não o único.

\section{Conclusão}

Nesta revisão sistemática foi possível identificar diferentes critérios de escolha portuária considerados pela perspectiva de cada autor, havendo significativa heterogeneidade entre os critérios avaliados como principais. Após a revisão dos resultados obtidos, conclui-se que a localização geográfica, citada em 71,4\% dos artigos selecionados, destaca-se como um critério essencial na escolha portuária, independentemente do tipo de amostra utilizado, pois se comprovou importante na seleção de portos para os três atores logísticos considerados (embarcadores, armadores e agentes de carga).

Percebe-se que são muitos os fatores que podem influenciar na seleção de portos. No entanto, a partir dos resultados da presente revisão, pode-se afirmar que, além da localização geográfica, os custos logísticos e o nível de serviço também são fatores importantes na escolha portuária.

A metodologia utilizada para analisar os critérios de escolha portuária, a identificação dos critérios mais importantes e o agrupamento desses critérios em categorias permitiram que se atingissem os objetivos do estudo. Para um resultado mais consistente, a classificação dos artigos selecionados nesta revisão sistemática foi realizada mais duas vezes. A metodologia mostrou-se eficiente e confiável e os resultados encontrados foram os mesmos nas diferentes rodadas de análise.

Por fim, conclui-se que a escolha portuária é uma decisão de elevada importância nos processos logísticos internacionais e que o sucesso nos processos de compra e venda pelos embarcadores (exportadores e importadores) pode ser influenciado pela boa ou má seleção de portos.

\section{Abstract}

With the globalization and development of international trade, competition between ports has intensified and port studies have been developed. Part of these studies addresses the issue of port selection criteria, considering the different actors involved in that choice. Given this context, this article presents an overview of the port selection criteria based on point of view of ship owners, 
cargo agents and shippers. The systematic review was the technique chosen because it allows a consistent and systematic analysis of the existing literature on the subject, explaining the main criteria for the selection of ports found in previous studies and identifying convergent and divergent aspects recorded on selected articles. The contribution of this systematic review of port choice criteria is based on the following aspects: identification of the main authors who studied the subject; leading institutions they are affiliated; major journals used for the presentation of research results; number of publications by country; number of publications by year; and identification of port selection criteria, considering the perspectives of the different actors involved.

Key-words: port choice; criteria; literature; systematic review.

\section{Referências}

BICHOU, K.; GRAY, R. A critical review of conventional terminology for classifying seaports. Transportation Research Part A: Policy and Practice, v. 39, n. 1, p. 75-92, 2005. crossref

CORDEIRO, A. M.; OLIVEIRA, G. M.; RENTERÍA, J. M.; GUIMARÃES, C. A. Revisão sistemática: uma revisão narrativa. Revista do Colégio Brasileiro de Cirurgiões, v. 34, n. 6, p. 428-431, 2007. crossref

D'ESTE, G. M.; MEYRICK, S. Carrier selection in a ro/ro ferry trade, part 1 - decision factors and attitudes. Maritime Policy and Management, v. 19, n. 2, p. 115-126, 1992. crossref

DE LA TORRE-UGARTE-GUANILO, M. C.; TAKAHASHI, R. F.; BERTOLOZZI, M. R. Revisão sistemática: noções gerais. Revista da Escola de Enfermagem da USP, v. 45, n. 5, p. 1260-1266, 2011. cross ref

GALVÃO, C. M.; SAWADA, N. O.; TREVIZAN, M. A. Revisão sistemática: recurso que proporciona a incorporação das evidências na prática da enfermagem. Revista Latino-Americana de Enfermagem, v. 12, n.3, p. 549-56, 2004 crossref

LEVY, Y.; ELLIS, T. J. Systems approach to conduct an effective literature review in support of information systems research. Informing Science Journal, v. 9, p.181-212, 2006.

LIMA, M. S.; SOARES, B. G.; BACALTCHUK, J. Psiquiatria baseada em evidências. Revista Brasileira de Psiquiatria, v. 22, n. 3, p. 142-146, 2000. crossref

LIRN, T. C.; THANOPOULOU, H. A.; BEYNON, M. J.; BERESFORD, A. K. C. An application of AHP on transhipment port selection: a global perspective. Maritime Economics and Logistics, v. 6, n. 1, p. 70-91, 2004.

MALCHOW, M. B.; KANAFANI, A. A disaggregate analysis of port selection. Transportation Research Part E: Logistics and Transportation Review, v. 40, n. 4, p. 317-337, 2004. cross ref

NIGHTINGALE, A. A guide to systematic literature reviews. Surgery, v. 27, n. 9, p. 381-384, 2009.

ONUT, S.; TUZKAYA, U. R.; TORUN, E. Selecting container port via a fuzzy ANP-based approach: A case study in the Marmara Region, Turkey. Transport Policy, v. 18, n. 1, p. 182-193, 2011. crossref

NIR, A. S.; LIN, K.; KIANG, G. S. Port choice behaviour - from the perspective of the shipper. Maritime Policy and Management, v. 30, n.2, p. 165-173, 2003. crossref

PIEPER, D.; BUECHTER, R.; JERINIC, P.; EIKERMANN, M. Overviews of reviews often have limited rigor: a systematic review. Journal of Clinical Epidemiology, v. 65, n. 12, p. 1267-1273, 2012. cross ref

SAMPAIO, R. F.; MANCINI, M. C. Estudos de revisão sistemática: um guia para síntese criteriosa da evidência científica. Revista Brasileira de Fisioterapia, v. 11, n. 1, p. 83-89, 2007. crossref

THOMAZ E SERRA, L.; MARTINS, R. S., BRONZO, M. O ambiente público-privado no processo de decisão quanto à operação portuária no Brasil: um estudo de caso. Revista Brasileira de Gestão de Negócios, v. 11, n. 31, p. 183-199, 2009. 
TONGZON, J. L. Determinants of port choice in a competitive environment. IAME Panama 2002 Conference Proceedings, 13-15 November, Panama, 2002

TONGZON, J. L. Port choice and freight forwarders. Transportation Research Part E: Logistics and Transportation Review, v. 45, n. 1, p. 186-195, 2009. crossref

TIWARI, P.; ITOH, H.; DOI, M. Shippers' port and carrier selection behaviour in China: a discrete choice analysis. Maritime Economics and Logistics, v. 5, n. 1. p. 23-39, 2003.

TOLLEY, R. S.; TURTON, B. J. Transport systems, policy and planning: a geographical approach. Singapore: Longman, 1995.

VALENTINE, V. F; GRAY, R. An organizational approach to port efficiency. IAME Panama 2002 Conference Proceedings, 13-15 November, Panamá, 2002.

VIEIRA, G. B. B.; PASA, G. S. Escolha portuária: um estudo piloto para a identificação de hub ports na costa brasileira através de análise conjunta e projetos de experimentos. Anais... XXV Encontro Nacional de Engenharia de Produção ENEGEP, Porto Alegre, 2005.

\section{APÊNDICE I - Artigos selecionados}

1. ACOSTA, M.; CORONADO, D.; DEL MAR CERBAN, M. Bunkering competition and competitiveness at the ports of the Gibraltar Strait. Journal of Transport Geography, v. 19, n. 4, p. 911-916, 2011. cross ref

2. BELOVIC, D.; MITROVIC, D. M. An approach to the selection of optimal transformation strategy in seaports. Promet - Traffic \& Transportation, v. 22, n. 3, p. 219-228, 2010.

3. BERGANTINO, A. S.; MUSSO, E. The role of external factors versus managerial ability in determining seaports' relative efficiency: an input-by-input analysis through a multi-step approach on a panel of Southern European ports. Maritime Economics and Logistics, v. 13, n. 1, p. 121-141, 2011. crossref

4. CHANG , Y. T.; LEE, S. Y. TONGZON, J. L. Port selection factors by shipping lines: Different perspectives between trunk liners and feeder service providers. Marine Policy, v. 32, n. 6, p. 877-885, 2008. crossref

5. CHANG, Y. T.; LEE, S. Y.; TONGZON, J. L. Port selection factors by shipping lines: Different perspectives between trunk liners and feeder service providers. Marine Policy, v. 32, n. 6, p. 877-885, 2008. cross ref

6. CHOU C. C. An empirical study on port choice behaviors of shippers in a multiple-port region. Marine Technology Society Journal, v. 43, n. 3, p. 71-77, 2009. crossref

7. CHOU, C. C. A fuzzy MCDM method for solving marine transshipment container port selection problems. Applied Mathematics and Computation, v. 186, n. 1, p. 435-444, 2007. crossref

8. CHOU, C. C. AHP model for the container port choice in the multiple-ports region. Journal of Marine Science and Technology, v. 18, n. 2, p. 221-232, 2010.

9. CHOU, C. C. An integrated quantitative and qualitative FMCDM model for location choices. Soft Computing, v. 14 , n. 7 , p. $757-77,2010$. cross ref

10. CHOU, C. C. Application of FMCDM model to selecting the hub location in the marine transportation: A case study in southeastern Asia. Mathematical and Computer Modelling, v. 51, n. 5-6, p. 791-801, 2010. cross ref

11. CHOU, C. C.; KUO, F. T.; GOU, R. H.; TSAI, C. L.; WONG, C. P.; TSOU, M. C. Application of a combined fuzzy multiple criteria decision making and optimization programming model to the container transportation demand split. Applied Soft Computing Journal, v. 10, n. 4, p. 1080-1086, 2010. crossref

12. DUCRUET, C.; ROZENBLAT, C.; ZAIDI, F. Ports in multi-level maritime networks: evidence from the Atlantic (1996-2006). Journal of Transport Geography, v. 18, n. 4, p. 508-518, 2010. crossref 
13. ESTACHE, A.; GONZÁleZ, M.; TRUjILlO, L. Efficiency Gains from Port Reform and the Potential for Yardstick Competition: Lessons from Mexico. World Development, v. 30, n. 4, p. 545-560, 2002. crossref

14. FERRARI, C.; PAROLA, F.; GATTORNA, E. Measuring the quality of port hinterland accessibility: The Ligurian case. Transport Policy, v. 18, n. 2, p. 382-391, 2011. crossref

15. GARCIA-ALONSO, L.; SANCHEZ-SORIANO, J. Port selection from a hinterland perspective. Maritime Economics and Logistics, v. 11, n. 1, p. 260-269, 2009. crossref

16. HARALAMBIDES, H.; VELDMAN, S.; VAN DRUNEN, E.; LIU, M. Determinants of a regional port-centric logistics hub: The case of East Africa. Maritime Economics and Logistics, v. 13, n. 1, p. 78-97, 2011. crossref

17. HEAVER, T. The Evolution and Challenges of Port Economics. Research in Transportation Economics, v. 16, n. 1, p. 11-41, 2003. crossref

18. LIRN, T. C.; THANOPOUlOU, H. A.; BEYNON, M. J.; BERESFORD, A. K. C. An Application of AHP on Transhipment Port selection: A Global Perspective. Maritime Economics and Logistics, v. 6, n. 1, p. 70-91, 2004. crossref

19. LOW, J. M. W.; LAM, S. W.; TANG, L. C. Assessment of hub status among Asian ports from a network perspective. Transportation Research Part A: Policy and Practice, v. 43, n. 6, p. 593-606, 2009. crossref

20. MUSSO, E.; FERRARI, C.; BENACCHIO, M. Port Investment: Profitability, Economic Impact and Financing. Research in Transportation Economics, v. 17, n. 1, p. 171-218, 2006. crossref

21. NG, A. S. F.; LIM, A. L. C.; LEONG, C. H.; CHENG, C. H. A competitiveness measurement framework for regional container hub ports: A case study in East Asia. International Journal of Logistics Systems and Management, v. 7, n. 3, p. 368-392, 2010. crossref

22. NG, K.Y. Assessing the attractiveness of ports in the North European container transhipment market: An agenda for future research in port competition. Maritime Economics and Logistics, v. 8, n. 3, p. 234-250, 2006. crossref

23. NOTTEBOOM T. An application of multi-criteria analysis to the location of a container hub port in South Africa. Maritime Policy and Management, v. 38, n. 1, p. 51-79, 2011. crossref

24. OLIVIER, D., SLACK, B. Rethinking the port. Environment and Planning A, v. 38, n. 8, p. 1409-1427, 2006. crossref

25. ONUT, S.; TUZKAYA, U. R.; TORUN, E. Selecting container port via a fuzzy ANP-based approach: A case study in the Marmara Region, Turkey. Transport Policy, v. 18, n. 1, p. 182-193, 2011. crossref

26. PJEVČEVIĆ, D.; VLADISAVLJEVIĆ, I.; VUKADINOVIĆ, K.; TEODOROVIĆ, D. Application of DEA to the analysis of AGV fleet operations in a port container terminal. Procedia - Social and Behavioral Sciences, v. 20, n. 1, p. $816-825,2010$.

27. RAVIBABU, M. A nested logit model of mode choice for inland movement of export shipments: A case study of containerised export cargo from India. Research in Transportation Economics, v. 38, n. 1, p. 91-100, 2013. crossref

28. RIMMER, P. J. Ocean liner shipping services: corporate restructuring and port selection/competition. Asia Pacific Viewpoint, v. 39, n. 2, p. 193-208, 1998. crossref

29. RODRIGUE, J. P.; NOTTEBOOM T. Foreland-based regionalization: Integrating intermediate hubs with port hinterlands. Research in Transportation Economics, v. 27, n. 1, p. 19-29, 2009. cross ref

30. SAEED, N. An analysis of carriers` selection criteria when choosing container terminals in Pakistan. Maritime Economics and Logistics, v. 11, n. 1, p. 270-288, 2009. crossref

31. SANCHEZ, R. J.; NG, A. K. Y., GARCIA-ALONSO, L. Port selection factors and attractiveness: The service providers' perspective. Transportation Journal, v. 50, n. 2, p. 141-161, 2011. crossref

32. SEO, J. S.; HA, Y. S. The Role of Port Size and Incentives in the Choice of Location by Port Users: A GameTheoretic Approach. The Asian Journal of Shipping and Logistics, v. 26, n. 1, p. 49-65, 2011. cross ref 
33. STEVEN, A. B.; CORSI, T. M. Choosing a port: An analysis of containerized imports into the US. Transportation Research Part E: Logistics and Transportation Review, v. 48, n. 4, p. 881-895, 2012. crossref

34. TANG, L.C.; LOW, J. M. W.; LAM, S. W. Understanding port choice behavior - A network perspective. Networks and Spatial Economics, v. 11, n. 1, p. 65-82, 2011. cross ref

35. TAVASSZY, L.; MINDERHOUD, M.; PERRIN, J. F.; NOTTEBOOM, T. A strategic network choice model for global container flows: specification, estimation and application. Journal of Transport Geography, v.19, n.6, p. 1163-1172, 2011. crossref

36. TIWARI P.; ITOH, H.; DOI, M. Shippers' Port and Carrier Selection Behaviour in China: A Discrete Choice Analysis. Maritime Economics and Logistics, v. 5, n. 1, p. 23-39, 2003. cross ref

37. TONGZON, J. L. Port choice and freight forwarders. Transportation Research Part E: Logistics and Transportation Review, v. 45, n. 1, p. 186-195, 2009. crossref

38. TONGZON, J. L. Determinants of port performance and efficiency. Transportation Research Part A: Policy and Practice, v. 29, n. 3, p. 245-252, 2012. cross ref

39. TONGZON, J., CHANG, Y. T.; LEE, S. Y. How supply chain oriented is the port sector? International Journal of Production Economics, v. 122, n. 1, p. 21-34, 2009. cross ref

40. UGBOMA, C.; UGBOMA, O.; OGWUDE, I. C. An analytic hierarchy process (AHP) approach to port selection decisions - Empirical evidence from Nigerian ports. Maritime Economics and Logistics, v. 8, n. 1, p. 251-266., 2006. cross ref

41. VELDMAN, S.; GARCIA-ALONSO, L.; VALLEJO-PINTO, J. A. Determinants of container port choice in Spain. Maritime Policy and Management, v. 38, n. 5, p. 509-522, 2011. crossref

42. WANKE, P. F., AFFONSO, C. R. Determinants of scale efficiency in the Brazilian 3PL industry. Produção, v. 21, n. 1, p. 53-63, 2011 crossref

43. WIEGMAnS, B. W.; HOEST, A.V. D.; NOTTEBOOM, T. E. Port and terminal selection by deep-sea container operators. Maritime Policy and Management, v. 35, n. 6, p. 517-534, 2008. crossref

44. WONG, W. G.; HAN, B. M.; FERREIRA, L.; ZHU, X. N. Factors influencing container transport: A fuzzy numberbased distribution model approach. Transportation Planning and Technology, v. 24, n. 3, p. 171-183, 2004.

45. WOO, S. H.; PETTIT, S. J.; KWAK, D. W.; BERESFORD, A. K. C. Seaport research: A structured literature review on methodological issues since the 1980s. Transportation Research Part A: Policy and Practice, v. 45, n. 7, p. 667-685, 2011. crossref

46. YAP, W. Y.; LAM, J. S. L. Competition dynamics between container ports in East Asia. Transportation Research Part A: Policy and Practice, v. 40, n. 1, p. 35-51, 2006. cross ref

47. YEO, G. T.; ROE, M., DINWOODIE, J. Evaluating the competitiveness of container ports in Korea and China. Transportation Research Part A: Policy and Practice, v. 42, n. 6, p. 910-921, 2008. cross ref

48. YEO, G. T.; ROE, M.; DINWOODIE, J. Measuring the competitiveness of container ports: logisticians' perspectives. European Journal of Marketing, v. 45, n. 3, p. 455-470, 2011. crossref

49. YEO, H. J. Competitiveness of Asian Container Terminals. The Asian Journal of Shipping and Logistics, v. 26, n. 2, p. 225-246, 2010. crossref

\section{Dados dos autores}

Nome completo: Guilherme Bergmann Borges Vieira

Filiação institucional: Universidade de Caxias do Sul - UCS - Rio Grande do Sul

Departamento: Centro de Ciências Sociais (CCSO) 
Função ou cargo ocupado: Professor e Pesquisador

Endereço completo para correspondência: Rua Francisco Getúlio Vargas, 1130 - CEP 95070-560 Caxias do Sul - RS

Telefones para contato: (51) 9944-8504

e-mail: gbbvieir@ucs.br

\section{Nome completo: Roberto Birch Gonçalves}

Filiação institucional: Universidade de Caxias do Sul - UCS - Rio Grande do Sul

Departamento: Centro de Ciências Sociais (CCSO)

Função ou cargo ocupado: Professor e Pesquisador

Endereço completo para correspondência: Rua Francisco Getúlio Vargas, 1130 - CEP 95070-560 Caxias do Sul - RS

Telefones para contato: (54) 8135-8590

e-mail:rbgoncal@ucs.br

\section{Nome completo: Fernanda Lazzari}

Filiação institucional: Universidade de Caxias do Sul - UCS - Rio Grande do Sul

Departamento: Centro de Ciências Sociais (CCSO)

Função ou cargo ocupado: Professor e Pesquisador

Endereço completo para correspondência: Rua Francisco Getúlio Vargas, 1130 - CEP 95070-560 Caxias do Sul - RS

Telefones para contato: (54) 8434-7651

e-mail: flazzar3@ucs.br

\section{Nome completo: Sarah Bertuol}

Filiação institucional: Universidade de Caxias do Sul - UCS - Rio Grande do Sul

Departamento: Centro de Ciências Sociais (CCSO)

Função ou cargo ocupado: Estudante graduada em Comércio Internacional

Endereço completo para correspondência: Rua Francisco Getúlio Vargas, 1130 - CEP 95070-560 Caxias do Sul - RS

Telefones para contato: (54) 8149-0779

e-mail: $\underline{\text { sarahbertuol@hotmail.com }}$

\section{Submetido em: 12/01/2014}

Aceito em: 22/10/2014 